\title{
Die Existenz, Abwesenheit und Macht des Wahnsinns. Eine kritische Übersicht zu Michel Foucaults Arbeiten zur Geschichte und Philosophie der Psychiatrie
}

\author{
Burkhart Brückner, Lukas Iwer und Samuel Thoma
}

Existence, Absence and Power of Madness: A Critical Review of Michel Foucault's Writings on the History and Philosophy of Madness

This article discusses Michel Foucault's main writings on "madness and psychiatry" from his early works up to the 1970s. On the one hand, we reconstruct the overall theoretical and methodological development of his positions over the course of the different periods in his oeuvre. On the other hand, we also take a closer look at Foucault's philosophical considerations regarding the subjects of his investigations. After an initial introduction of our conceptual approach, we draw on the most recent research on Foucault to show to what extent the phenomenological description of the topic at hand and the historical-critical perspective that are reflected in his early writings of 1954 (the Introduction to Binswanger's Dream and Existence and Mental IIIness and Personality) laid the ground for his later work. Moving on to Foucault's work during the 1960s, we look at the core features and methodological bases of his 1961 classic Folie et déraison (History of Madness). His propositions regarding the "absence of madness" in modernity are conceptualized as an inherently contradictory attempt to liberate the topic under study from the common assumptions at that time. We then situate his 1973/74 lectures on Psychiatric Power in the context of his shift towards analyzing the dynamics of power and highlight the renewed shift of focus in his statements on the "productivity" of madness as an effect of power. Finally, we sum up our critique by taking into account the history of the reception of Foucault's writings and ask about their potential significance for the contemporary philosophy and history of psychiatry.

Keywords: Michel Foucault, Madness, Psychiatry, History, Oeuvre, Critique

In diesem Artikel diskutieren wir Michel Foucaults Hauptwerke zum Thema "Wahnsinn und Psychiatrie" von den Frühschriften bis in die siebziger Jahre. Zum einen rekonstruieren wir die globale theoretische und methodologische Entwicklung seiner Positionen im Lauf der verschiedenen Werkperioden. Zum anderen arbeiten wir Foucaults philosophische Überlegungen zum Gegenstand seiner Untersuchungen heraus. Nach der einleitenden Problemstellung zeigen wir entsprechend der neueren Forschung, inwiefern Foucaults frühe Positionen von 1954 (in der Einführung zu Binswangers Traum und Existenz sowie in Geisteskrankheit und Persönlichkeit) das spätere Werk durch eine phänomenologische Deskription des Gegenstands und einen historisch-kritischen Anspruch vorbereitete. Davon ausgehend thematisieren wir die zentralen inhaltlichen und methodologischen Prämissen in Foucaults Wahnsinn und Gesellschaft von 1961. Seine Thesen zur "Abwesenheit des Wahnsinns" in der Moderne verstehen wir als einen in sich widersprüchlichen Versuch, den Gegenstand der Untersuchung von gängigen Vorannahmen zu befreien. Anschließend situieren wir Foucaults Vorlesungen Die Macht der Psychiatrie von 1973/74 im Kontext seiner machtanalytischen Werkphase und betonen seinen erneuten Standpunktwechsel in den Annahmen zur "Produktivität" des Wahnsinns als Machteffekt. Abschließend resümieren wir unsere Kritik im Zusammenhang mit der Rezeptionsgeschichte der dargestellten Studien und fragen nach 
Die Geschichte des Wahnsinns und der Psychiatrie nimmt eine Schlüsselposition in Michel Foucaults philosophischer Historiographie ein. Kein anderes Thema hat Foucault zwischen 1954 und 1974 so häufig wieder neu aufgegriffen und weiter entwickelt. Die Rezeption konzentrierte sich bislang auf seine Arbeiten der sechziger Jahre, eine kritische Gesamtschau stand aus. ${ }^{1}$ Wir beginnen die Rekonstruktion dieser Werklinie mit zwei frühen Publikationen von 1954, der Einführung zu Ludwig Binswangers Studie Traum und Existenz sowie der Monographie Geisteskrankheit und Persönlichkeit. Anschließend behandeln wir das thematische Hauptwerk Wahnsinn und Gesellschaft von 1961 und darauf aufbauend die Vorlesungen Die Macht der Psychiatrie von 1973/74. Wir beachten auch kleinere Schriften sowie Interviews und fragen zunächst, wie Foucault seine Positionen parallel zur allgemeineren Entwicklung seines Werks entfaltete: Was verstand er unter "Geisteskrankheit" oder „Wahnsinn“? In welchem Verhältnis stehen dazu die Medizin und die Psychiatrie? Dann fragen wir nach der epistemischen Basis dieses Gegenstandsverständnisses: Auf welcher philosophischen Grundlage und mit welchen Prämissen nahm er sein historisches Objekt wahr?

Einige Anhaltspunkte für die Rekonstruktion geben die in der Forschung geläufigen Systematisierungen des Gesamtwerks. So unterschieden Hubert L. Dreyfus und Paul Rabinow (1987 [1982]) eine Phase der Diskursanalyse und eine Phase der Machtanalytik, während Gilles Deleuze (1987) die inhaltlichen Schwerpunkte „Wissen“, „Macht“ und „Subjektivierung“ betonte. Hinrich Fink-Eitel (1997) sah vier Werkphasen: das Frühwerk der fünfziger Jahre, die Archäologie der sechziger Jahre, die Genealogie der siebziger Jahre, sowie die Subjekttheorie der achtziger Jahre. Kritisch gegenüber solchen Periodisierungen verwies Clemens Kammler (2008: $9 \mathrm{f}$.) zu Recht auf Foucaults ständige „Verlagerungen der Gegenstandsfelder“, „Transformationen der Verfahren“ und begriffliche Innovationen. Genau solche typischen Verschiebungen und Wechsel der Perspektiven lassen sich am Thema „Psychiatrie und Wahnsinn“ exemplarisch aufzeigen.

Um 1954 beschäftigte Foucault sich mit der Phänomenologie, dem Marxismus und der Psychoanalyse, um ein anthropologisch-existenzielles Modell der "Geisteskrankheiten“ zu entwerfen. Eine fundamentale Wende ergab sich 1961 in Wahnsinn und Gesellschaft unter dem Einfluss von Georges Canguilhem, Friedrich Nietzsche und Jacques Lacan mit einer Theorie der 
Sprachlichkeit des Wahnsinns, die Foucault ein Jahr später in der Monographie Raymond Roussel von 1963 konkretisierte. Nach methodologischen Klärungen in Die Ordnung der Dinge (1966) und in der Archäologie des Wissens (1969) bereitete er ab 1973 den Übergang zur Analytik der Macht in den Vorlesungen Die Macht der Psychiatrie vor. Seitdem behandelte er die Institution "Psychiatrie“ und die Gruppe der „Wahnsinnigen“ gleichrangig neben weiteren Institutionen (z. B. dem Gefängnis) und anderen sozial marginalisierten Gruppen (z. B. Homosexuellen). Seine Arbeiten ab 1976 zur Sexualität, zur antiken Selbstsorge, zur Biopolitik oder Gouvernementalität berühren unser Thema nicht mehr. Die anvisierte Werklinie spiegelt also die methodologischen Kurswechsel von der Phänomenologie der fünfziger Jahre über die Archäologie und Diskursanalyse der sechziger bis hin zur Genealogie und Machtanalytik der siebziger Jahre. Dabei veränderten sich wiederum in jeweils typischer Weise Foucaults Modelle des historischen Zusammenhangs zwischen den Phänomenen des Wahnsinns und dem Gegenstandsbereich der Psychiatrie.

So führt die Frage nach dem Gegenstand von Foucaults Untersuchungen zu seinen unterschiedlichen Auffassungen über die historische Konstitution und Identität eines Gegenstandsfelds. Der Begriff des „wissenschaftlichen Gegenstands“ wurde ihm spätestens mit der Archäologie des Wissens von 1969 fragwürdig. Seitdem verstand sich Foucault (1977 [1971]: 32 f.) als Theoretiker des Diskontinuierlichen im Rahmen einer als „seriell“ begriffenen Historizität von Ereignissen und verwarf die traditionelle Ideengeschichte als illusionären Effekt der Subjektbegriffe, die ihr zugrundliegen würden. Die Phänomene der „Geisteskrankheiten“ begriff er demnach in den siebziger Jahren nicht mehr als konsistenten wissenschaftlichen Gegenstand, sondern analysierte sie als einen Schwarm von aufeinander bezogenen Aussagen im konstitutiven Zusammenhang des psychiatrischen Dispositivs der Macht.

Wir können also Foucaults Positionen nicht als einheitliche Formationen rekonstruieren, sondern müssen die Übergänge und Widersprüche seiner Theoriebildung im Kontext der Kategorien „Geisteskrankheit“, „Wahnsinn“ und „Psychiatrie“ herausarbeiten. In diesem Sinne halten wir es für legitim, von einem thematisch eingrenzbaren, aber konzeptionell differierenden Bereich eines „Gegenstandes“ zu sprechen, dessen jeweilige Konstruktion, Prämissen und Logik im Werkverlauf kritisch aufgewiesen werden können. Dabei bleibt der sozialhistorische Kontext von Foucaults Theoriebildung ebenso im Hintergrund wie seine mögliche persönliche Nähe zum Thema und ihre Verarbeitung im Werk (siehe dazu Eribon 1999 [1989]: 53-61, 2016 [2009]: 213-219). 


\section{Anthropologie des Ausdrucks, der Existenz und des Wahnsinns}

Foucaults Arbeiten zwischen 1954 bis 1960 wurden lange marginalisiert und erst ab den achtziger Jahren wiederentdeckt. ${ }^{2}$ Auch wir betonen ihre Eigenständigkeit. Zwei Texte aus dem Jahr 1954 interessieren uns hier besonders: die Einführung zur französischen Übersetzung von Binswangers 1930 publiziertem Aufsatz Traum und Existenz sowie Foucaults erste Monographie Maladie mentale et personnalité (Geisteskrankheit und Persönlichkeit).

Die Einführung in Binswangers Traum und Existenz

1954 entwickelte Foucault in seiner Einführung zu Binswangers Traum und Existenz eigene Positionen zu Kategorien wie „Menschsein“, „Traum“, „Imagination“ und „Ausdruck“. Der Mensch begegne im Traum, in der Imagination und im Wahnsinn fundamentalen Strukturen seiner Existenz, die sich der Verfügung der Vernunft und den objektiven diskursiven Strukturen entzögen. Allerdings müsse, um dies zu zeigen, erst der innere Sinn der Bildersprache des Traums angemessen übersetzt werden. Foucault (2001[1954]: 117) verwies auf Sigmund Freuds Traumdeutung (1900), Melanie Kleins Arbeiten über frühkindliche Phantasmen und Lacans Thesen zum Verhältnis von Sprache (langage) und Sprechen (parole). Doch die Psychoanalyse scheitere, da sie einen theoretisch konstruierten Sinngehalt unter die Bildersprache des Traumes lege, ihm „Formen des wachen Bewusstseins“ zuschreibe und so ,jedes Privileg als besondere Erfahrungsform" nehme (Foucault 2001 [1954]: 126, 118). Um der Erfahrung als solcher gerecht zu werden, beschrieb Foucault (ebd.: 119) den fundierenden Ausdrucksakt, der dem Sprechen vorausginge:

Wenn eine Person spricht, verstehen wir, was sie sagt, nicht nur durch das signifikative Erfassen der Worte, die sie gebraucht und der Strukturen von Sätzen [...], sondern wir lassen uns auch von der Melodie der Stimme leiten, die hier nachgibt und zu zittern beginnt, dort jedoch jene Festigkeit und jene Erregung annimmt, an der wir den Zorn erkennen.

Sinn habe also nicht nur das, „was“ gesagt werde, sondern auch, „wie“ es gesagt werde. Dementsprechend interpretierte er das Träumen, die Imagination und den Wahnsinn als Formen eines „existenziellen“ Ausdrucks und individuellen Seinsstils mit drei zentralen Argumenten.

Erstens besitze der Traum einen anthropologisch bedeutsamen Wahrheitswert ohne versteckten, inhaltlichen Sinn. Vielmehr handele es sich um eine Erfahrungsform, in der sich dem Menschen der Gang seiner Existenz offenbare: Im Träumen erfahre sich der Mensch als „im verlassenen Raum 
sich aushöhlende, im Chaos sich brechende, im Lärm auseinanderfliegende, sich als kaum mehr atmendes Tier in den Netzen des Todes verfangende Existenz" (ebd.: 151). Foucaults Begriff der Freiheit muss hier im Sinne einer verneinenden „Befreiung“ von den Regeln des Wachbewusstseins begriffen werden (ebd.: 126-130) - was unter verändertem Vorzeichen auch wieder in Wahnsinn und Gesellschaft zum Tragen kam. Zum zweiten verstand Foucault die „wache“ Imagination als einen besonderen Modus, der nicht an den „Normen der objektiven Wahrheit“ messbar sei (ebd.: 168). Der Mensch erlebe dabei vielmehr eine Verwandlung seiner Seinsweise, in der er gerade - anders als Jean-Paul Sartre (1971 [1940]: 279-285) behauptete - als selbstbestimmtes, aktiv imaginierendes Subjekt verschwinde und der Ausdruckswelt ausgeliefert sei (Foucault 2001 [1954]: 164f.). Drittens verdeutlichte Foucault seine Thesen am Beispiel der Psychiatrie mit Binswangers Patientin Ellen West, die sich 1921 das Leben nahm (vgl. Akavia 2008). Foucault (2001 [1954]: 157-162) schloss sich Binswangers (1945) nachträglicher daseinsanalytischer Interpretation an: West habe ihre Welt als unkontrollierbares Pendeln zwischen zwei gegenteiligen Weltentwürfen empfunden. Im Zentrum ihrer Erfahrung stehe das tragische Moment der schicksalhaften Ambivalenz ihrer Existenz zwischen einer ätherischen Gedankenwelt des "Aufstiegs“ und einer Welt des ausweglosen „Falls“. Dies habe schließlich zu ihren „wahnhaften Erfahrungen vom ,Ende der Welt““ geführt. $^{3}$

Foucaults Text enthielt einige für sein weiteres Werk wichtige Motive. Thematisch zeigte er ein noch ausdruckspsychologisches Interesse für präverbale Erfahrungen und das „tragische“ Moment im Wahnsinn. Philosophisch gesehen entwickelte er mit seinen Kerngedanken über die Seinsweise der Existenz eine kategoriale Differenz zwischen der von ihm beabsichtigten "Anthropologie des Ausdrucks" (ebd.: 174) und dem orthodoxen strukturalistischen Sprach- und Diskursbegriff auf der Basis einer lacanianisch inspirierten Lesart der Psychoanalyse (ebd.: 117 f.). Zwar gestand er gemäß der traditionellen strukturalistischen Unterscheidung zwischen Sprechen (parole) und Sprache (langue) auch dem Traum die Struktur einer langue zu (ebd.: 114). Jedoch sei diese sprachliche Struktur eben die eines "Ausdrucksgeschehens“, das sich jeder diskursiven und „logischen Analyse des Ganzen" entziehe, in deren Rahmen auch die Psychoanalyse noch operiere (ebd.: 114, 126). Die strukturalistische Annahme, im sprachlichen Referenzsystem würden Zeichen und Bezeichnetes auseinandertreten - wie etwa das Wort „Zorn“ und der Zorn selbst, gelte also nicht für die Ausdrucksphänomene der Existenz, bei denen etwa der Ausdruck des Zorns und der Zorn selbst ineinander fielen (vgl. ebd.: 120). Der Ausdrucksakt könne daher weder methodisch noch konzeptionell im Zeichensystem der Sprache und des Diskurses aufgelöst werden. Dabei orientierte Foucault 
sich philosophisch weniger an Edmund Husserls Bewusstseinsphänomenologie oder an Sartres Philosophie der Freiheit, sondern an Binswangers Daseinsanalyse und Maurice Merleau-Pontys Phänomenologie des Leibs und der Wahrnehmung (vgl. Basso 2016a). Mit der kritischen Aneignung dieser Autoren fasste Foucault die Idee eines Wesens, einer Struktur oder eines Existenzials nicht als etwas der konkreten Erfahrung und dem Fall Vorgeordnetes oder Zugrundeliegendes auf, sondern als die immanente Struktur des Gegebenen selbst. Diesen Gedanken eines der Existenz eigentümlichen, konkreten Ausdruckssinns gab er zwar im weiteren Werk auf, aber entwickelte die Idee einer Erfahrung, die sich der Logik des Diskurses entzieht, in Wahnsinn und Gesellschaft auf allgemeinerer Ebene weiter.

Phänomenologie der Persönlichkeit und materialistische Psychologie In seinem ersten Buch Geisteskrankheit und Persönlichkeit von 1954 konfrontierte Foucault seine Thesen über die Existenz und ihren besonderen, immanenten Ausdruckssinn mit evolutionsbiologischen und psychoanalytischen Ansätzen und entwickelte ein historisch-materialistisches Verständnis der Psychiatrie und ihres Gegenstandes. ${ }^{4}$ Die damit verbundene Hinwendung zur Theorie der Persönlichkeit ist nach Elisabetta Basso (2016b: 34-41) im Kontext der damaligen französischen Debatten um ein konkretes und ganzheitliches Verständnis der Psyche zu sehen. Foucaults Rekurs auf den Persönlichkeitsbegriff überkreuzte sich im Text vielfach mit Bestimmungen der menschlichen Existenz und existenziellen Strukturen. Der Begriff der Existenz ist damit unserer Ansicht nach das verbindende Konzept zwischen Foucaults beiden hier thematisierten Publikationen.

Einleitend äußerte Foucault (1954: 3-17) sich in Geisteskrankheit und Persönlichkeit kritisch zum psychiatrischen Verständnis von Krankheit und Diagnostik. Der traditionelle diagnostische Blick isoliere Einzelsymptome und führe sie reifizierend auf organische Störungen zurück. Demgegenüber entfaltete Foucault eine eigenständige phänomenologische Psychopathologie der biographisch verstehbaren Persönlichkeit. Die Persönlichkeit sei „das Element, in dem sich die Krankheit entwickelt, und das Kriterium für deren Beurteilung; sie ist zugleich die Wirklichkeit und das Maß der Krankheit" (ebd.: 10). Sie verleihe nicht nur jedem einzelnen Symptom einen kohärenten Seinsstil (ebd.: 13), sondern gehe auch jeder möglichen Determination durch vergangene oder unbewusste Entwicklungsstadien voran, mögen diese nun physiologisch oder psychoanalytisch konzipiert sein (ebd.: 23-35). Den konkreten Seinsstil der Persönlichkeit bezeichnete Foucault (ebd.: 52) auch als das „a priori der Existenz“. Damit forderte er eine Wissenschaft, die weniger unbewusste Dynamiken untersucht, sondern vielmehr die konkret existierende Persönlichkeit, auf die diese sich beziehen: „Die Wissenschaft der Psychopathologie kann nur die Wissen- 
schaft von der kranken Persönlichkeit sein“ (ebd.: 34). Dafür müsse man sich mittels einer „Phänomenologie der Geisteskrankheit“ in das „Zentrum dieser Erfahrung“ versetzen (ebd.: 56). Angelehnt an Binswanger definierte Foucault das Pathologische als Verlust der Bezogenheit auf Gemeinschaft (ebd.: 68). Diesen Rückzug in den „idion kosmon“ der Selbstbezüglichkeit erlitten die Betroffenen wie ein „äußeres Schicksal“, dem sie hilflos ausgeliefert seien (vgl. Binswanger 1994 [1930]: 113). Aber die Kategorie der Persönlichkeit erschöpfte sich für Foucault nicht in einer pathologisch wandelbaren, existenziellen Struktur, sondern beinhaltete auch ein bewusstes Selbstverhältnis:

Das Bewußtsein, das der Kranke von seiner Krankheit hat, ist absolut original. Sicher ist nichts so falsch wie der Mythus von der Krankheit, die nichts von sich selbst weiß [...]. Es ist nicht so, als stünde der Arzt auf der Seite der Gesundheit, die alles Wissen über die Krankheit besitzt, und der Kranke auf der Seite der Krankheit, die nichts von sich selbst weiß, nicht einmal ihre Existenz (Foucault 1954: 56).

Das Selbstbewusstsein des Individuums lasse sich also weder von den tiefgreifenden Wandlungen der Erfahrungswelt noch vom medizinischen Zugriff vereinnahmen.

Sieht man den Begriff der Existenz als Leitbegriff von Foucaults ersten beiden Schriften, ergab sich 1954 eine dreifache konzeptionelle Abgrenzung: Er schützte den Existenzbegriff vor biologistischen Reduktionismen, verteidigte ihn gegenüber diskurstheoretischen Vereinnahmungen und hob das Selbstbewusstsein des Individuums vom Wahnsinn ab. Diese Ideen überführte er anschließend in eine materialistische Gesellschaftstheorie der Entfremdung (ebd.: $89 \mathrm{f}$.). Denn die zuvor analysierte „Subjektivität des Verrückten“ (ebd.: 69) könne nur von den „äußeren und objektiven Bedingungen“ (ebd.) der Welt her begriffen werden. Eine „Geisteskrankheit" galt ihm nun als individueller Ausdruck eines entfremdenden gesellschaftlichen Kontexts:

[W]enn der Mensch dem, was in seine Sprache eingeht, fremd bleibt, wenn er an dem, was seine Tätigkeit hervorbringt, keine lebendige menschliche Bedeutung mehr erkennen kann, wenn die ökonomischen und sozialen Bestimmungen ihren Zwang tun [...], dann lebt er in einer Kultur, die eine pathologische Form wie die Schizophrenie möglich macht (Foucault 1954: 89).

Daraus folgte ein direkt praxisbezogenes Verständnis der Psychologie: „Die wahre Psychologie [...] muss, wie jede Wissenschaft des Menschen, zum Ziel haben, seine Entfremdung aufzuheben“ (ebd.: 110). Umgesetzt sah Foucault diese Überlegung ,in einer Strukturreform der medizinischen 
Versorgung und der psychiatrischen Krankenhäuser“ und erklärte mit Blick auf frühe sozialpsychiatrische Initiativen: „Eine solche Reform wird bereits von bestimmten Ärzten eingefordert und begonnen" (ebd.: 109). Im neuen, politisch konnotierten Konzept einer Theorie der Entfremdung entfiel allerdings der phänomenologische Persönlichkeitsbegriff. Kommentarlos entwarf Foucault ein materialistisches Konzept der Persönlichkeit in Anlehnung an Iwan Petrowitsch Pawlows (1849-1936) reflexpsychologische Theorie der höheren Nerventätigkeit (ebd.: 91-110). Die Gründe für diesen Perspektivwechsel liegen offenbar in dem wissenschaftlichen Milieu, zu dem er sich hingezogen fühlte. Intensiv rezipierte Foucault die 1951 von dem Entwicklungspsychologen Henri Wallon (1879-1962) gegründete Zeitschrift La raison. Cahiers de psychopathologie scientifique (vgl. Paltrinieri 2015: 201-205). In diesem Forum der historisch-materialistisch orientierten Reformpsychiater wurde Pawlows Ansatz als eine mit dem Marxismus und dem Konzept der Entfremdung durchaus vereinbare, objektive Psychologie empfohlen, während die phänomenologische Psychiatrie als unwissenschaftlich galt (ebd.: 208-212).

Wir sehen also Foucault Ende der fünfziger Jahre gespalten in seinem Interesse an der phänomenologischen Psychiatrie einerseits und einer materialistischen Psychologie andererseits. Er überwand das Dilemma mit einem epistemisch radikalen, an Canguilhem, Lacan und Nietzsche angelehnten Perspektivwechsel in Wahnsinn und Gesellschaft. Davon zeugte auch die zweite, überarbeitete und 1962 publizierte Auflage von Geisteskrankheit und Persönlichkeit unter dem Titel Psychologie und Geisteskrankheit (Foucault 1968 [1962]). Diese Version des Buches ersetzte die erste Auflage und ließ den Text nun so aussehen, als habe Foucault bereits 1954 sein Konzept von 1961 ohne Rekurs auf Pawlow, die Anthropologie oder die Entfremdungstheorie antizipiert. Denn diese Neuauflage enthielt einen inhaltlichen Bruch: Foucault wollte nunmehr weder die innere Erfahrung (sensu Phänomenologie) noch deren Entfremdung (materialistische Psychologie) untersuchen, sondern fragte nach den geschichtlichen, soziokulturellen und wissensphilosophischen Voraussetzungen - dem „historischen Apriori“ - von Subjektivität und der vermittelnden Rolle der Psychologie und Psychiatrie (vgl. May 2005: 302-308; Macherey 1986). ${ }^{5}$

Dennoch sehen wir in diesem Bruch auch eine bedeutsame Kontinuität: Wie Basso (2012) gezeigt hat, übertrug Foucault im Übergang vom „existenziellen Apriori“ zum neuen „konkreten“ oder „historischen Apriori“ die Idee einer sich am „Wie“ des Gegebenen selbst zeigenden, kohärenten Struktur der Erfahrung, die sich nicht aus universalen Gesetzen deduzieren lasse, auf das generelle Verständnis von Historizität. Demnach seien historische Aussagensysteme nicht durch formale Mechanismen oder Regeln zu charakterisieren, sondern durch die „innere Logik“ (Foucault 1969 [1961]: 
254), die sie miteinander verbinde. Diese „innere Logik“ sei jeweils als eine konkrete historische Struktur zu bestimmen, ohne an ein übergreifendes Gesetz der Geschichte oder der Wahrheit gebunden zu sein.

\section{Das Schweigen der Unvernunft}

1961 erschien Foucaults Dissertation Folie et déraison. Histoire de la folie à l'âge classique (dt. Wahnsinn und Gesellschaft. Eine Geschichte des Wahns im Zeitalter der Vernunft). ${ }^{6}$ Sie umfasste die Epoche der französischen Klassik zwischen dem 17. und 18. Jahrhundert samt einem Vorspiel zur Renaissance und einem Ausblick ins 19. Jahrhundert. Hatte Foucault zuvor noch an einem positiven, in der Phänomenologie verankerten Begriff der "Geisteskrankheit“ festgehalten, positionierte er diesen nun fundamental neu gegenüber dem Begriff des „Wahnsinns“. Während die „Geisteskrankheiten“ als diskursive Phänomene historisch abbildbar seien, besitze der „Wahnsinn“ grundsätzlich eine gegenüber der subjektiven Erfahrung wie auch gegenüber theoretischen Systemen neutrale, „abwesende“ Position. Der Wahnsinn entziehe sich den diskursiven Optionen, ihn zu erfassen. Diese gäben zwar Auskunft über die Gestalten der Geisteskrankheit, aber nicht über den Wahnsinn selbst, dessen Ausdruck höchstens noch in literarischer Sprache überdauere. Die Denkfigur von der „Abwesenheit des Wahnsinns" führte Foucault zu einer konsequenten Kritik des modernen Begriffs vom Menschen, dem eine „Leere“ eigen sei, deren Reflexion die Brüchigkeit und Endlichkeit aller Anthropologie zeige. Wie aber verankerte er diese Thesen am historischen Material und welche epistemischen Prämissen erforderte das Projekt?

\section{Das Problem des historischen Objekts}

Im Vorwort von Wahnsinn und Gesellschaft erklärte Foucault (1969 [1961]: 11), er wolle „im Lichte der großen nietzscheanischen Forschungen die Dialektik der Geschichte mit den unbeweglichen Strukturen der Tragik konfrontieren." Damit setzte er den früheren Gestus der Kritik fort, um das Problem der historischen Identität seines Gegenstandes einzuebnen: Die „Sprache der Psychiatrie“ und der „Monolog der Vernunft über den Wahnsinn“ seien zu überwinden, da sie den Wahnsinn im Lauf der Moderne zum „Schweigen“ gebracht hätten. Nun ginge es um die Geschichte „des Wahnsinns selbst“ im Sinne einer "Archäologie dieses Schweigens" (ebd.: 8). Foucault verzichtete auf jede „Finalität der Erkenntnis“ und „Orthogenese“ der traditionellen Fortschrittsgeschichte, um das „konkrete Apriori“ der modernen Psychopathologie und damit die epistemologischen und histo- 
rischen Möglichkeitsbedingungen des Begriffs der „Geisteskrankheit“ herauszuarbeiten (ebd.: 116, 126). Präsentistische Ansätze schieden demnach aus, ${ }^{7}$ aber die gegenstandsbezogene Frage blieb: „Was also ist der Wahnsinn in seiner allgemeinsten, aber konkretesten Form für denjenigen, der von Anfang an jede Ingriffnahme des Wahnsinns durch die Wissenschaft ablehnt? Wahrscheinlich nichts anderes als das Fehlen einer Arbeit [absence d'œuvre]“. ${ }^{8}$ Die Bestimmung des Wahnsinns als „abwesendes Werk“ zielte keineswegs auf die pathologische Unproduktivität konkreter Subjekte, sondern beruhte auf einem neuen epistemischen Modell. Nicht mehr die Existenz des Menschen sei primär, sondern die vorgängige Struktur der Sprache. Basso (2016b: 42 f.) hat demgemäß auf Lacans Einfluss hingewiesen. Jacques Lacan (1997 [1955/56]: 230, 179) ging in den fünfziger Jahren von der Sprachlichkeit der psychischen Realität aus und konzipierte die Psychosen als radikalen Bruch mit dem Symbolischen: Das Subjekt antworte auf eine „Abwesenheit des Signifikanten“ mit der Entwicklung manifester Wahnideen, nachdem es diesen selbst „in die äußere Finsternis“ verworfen habe. Mit einem ähnlichen, auf die historische Ebene verlagerten Konzept erschien Foucault (2001 [1964]: 547) die Erfahrung des Wahnsinns nun als „Schweigen“ ohne Rückhalt in der Struktur eines Sinns. Dieses Schweigen halte vielmehr jeden Sinn zurück und in der Schwebe, sei „leer“ und reine Potentialität ohne Werk. Der Bezug auf einen sich entziehenden Gegenstand prägte auch Foucaults künftige Theorie der Literatur in Anlehnung an Maurice Blanchot, Georges Bataille oder Raymond Roussel („Abwesenheit des Autors") ebenso wie die Archäologie des Wissens von 1969.9 In Wahnsinn und Gesellschaft griff Foucault (1969 [1961]: 245 f.) somit auf die 1954 entwickelte Kritik des orthodoxen Strukturalismus zurück: Wie der Traum habe der Wahnsinn eine gegenüber den Diskursen autonome, sprachliche Qualität - aber nicht mehr als existenzielles „Ausdrucksgeschehen", sondern als Strukturprinzip der Erfahrung.

Dieses Modell zog gravierende inhaltliche Verschiebungen nach sich: Ein primärer „Einschnitt“ trenne das "Gemurmel einer Sprache, die von allein spricht" (ebd.: 12, Hervorhebung im Original) von allen folgenden kulturellen Sinngehalten als Voraussetzung der Historiographie: „Jene Erfahrungsstruktur des Wahnsinns, die völlig von der Geschichte abhängt, die aber an ihren Grenzen und dort ruht, wo diese sich entscheidet, ist der Gegenstand dieser Untersuchung." (ebd.: 8, 13). ${ }^{10}$ Der Begriff der Erfahrung nahm hier für Foucault den systematischen Ort ein, den später die Episteme oder das Dispositiv besetzen sollten. Erfahrung galt also in erster Linie als geschichtlich-kulturelle Grenzerfahrung, die zugleich eine Ursprungsstruktur impliziere, welche sich der Repräsentation entzieht, aber diese auch überdauere und wirksam bleibe (Unterthurner 2007: 150). Diese Erfahrung sei allerdings empirisch nicht zugänglich, der „Staub jener 
konkreten Schmerzen, jener unsinnigen Worte" der Wahnsinnigen könne nicht rekonstruiert werden (Foucault 1969 [1961]: 11, 13). Die „ursprüngliche Reinheit" ihrer Erfahrung bliebe verborgen. So enthielt Wahnsinn und Gesellschaft kaum Aussagen der „Irren“ selbst. ${ }^{11}$ Die Idee einer "history from below“, gestützt auf Selbstzeugnisse, wie sie etwa der britische Historiker Roy Porter (1985) entwickelte, schien unmöglich. Anstatt solche Dokumente in den Archiven zu suchen, wie es vielleicht noch seine vormaligen Arbeiten nahegelegt hätten, untersuchte Foucault vorrangig offiziöse Quellen und Lehrbuchtexte.

Die sprachtheoretische Begründung dieser Haltung präzisierte er in der 1963 erschienenen Studie über den Dichter Raymond Roussel (1877-1933), ein Patient von Pierre Janet. Roussels literarische Methoden der Produktion von Geschichten aus phonetischen Verschiebungen und der abweichenden Bedeutung homophoner Wörter oder Sätze verstand Foucault (1989 [1963]: 64) als „Sprachmaschinen“: „Jedes Wort ist zugleich belebt und gefährdet, erfüllt und entleert durch die Möglichkeit, daß es da noch ein zweites Wort gäbe - dieses oder jenes oder weder das eine noch das andere, sondern ein drittes oder nichts." (ebd.: 18). Der Signifikant bezeichne also nie eindeutig das Signifikat. So entstehe Sprache in ihrer Fülle immer aus dieser Differenz heraus durch einen Mangel an Worten. Wie der Tod für die Medizin und der Wahnsinn für die Psychiatrie sei diese „Leere“ konstitutiv für die Literatur - ohne vorbestimmte Struktur, ohne ordnendes Prinzip, ohne festen Sinn.

Mit dieser epistemischen Prämisse, die als ontologische oder transzendentale Prämisse gedeutet worden ist (Mazumdar 2008; Unterthurner 2007), ergab sich in Wahnsinn und Gesellschaft für die historische Arbeit ein tertium comparationis, das den Vergleich zwischen Quellen und Gegenstandsverständnis leitet, lediglich am Maßstab jener angeblichen Opposition zwischen Sinn und Nicht-Sinn, um ,jenen Punkt Null der Geschichte des Wahnsinns wiederzufinden [...], an dem der Wahnsinn noch undifferenzierte Erfahrung, noch nicht durch eine Trennung gespaltene Erfahrung ist" (Foucault 1969 [1961]: 7). Diese Setzung einer Einheit von Vernunft und Wahnsinn bezeichnete Foucault als „Unvernunft“ und im Kern „tragisch“, während die Ereignisse des manifesten „Wahnsinns“ im Lauf der Geschichte stetig als Formen der "Geisteskrankheit" registriert worden seien (ebd.: 370, 368-380). Der medizinisch-psychologische Zugriff verfehle seit dem Ende der Renaissance den tragischen Kern des Wahnsinns, obwohl dieser sich zuweilen noch literarisch auskristallisiere (etwa bei Donatien-Alphonse-François de Sade, Friedrich Hölderlin, Gérard de Nerval, Vincent van Gogh, Antonin Artaud; ebd.: 9, 350-357, 536). Wie verankerte Foucault dieses Motiv im historischen Material? 
Tragischer Wahnsinn und geblendete Vernunft

1954 war die personale Tragik in der Erfahrung des Wahnsinns noch mit den Metaphern des Aufstiegs, der Krisis und des Falls verbunden. Der Wahnsinn sei unerreichbar für das Wachbewusstsein und zugleich ursprünglicher Ausdruck der Freiheit der Existenz. 1961 übertrug Foucault (1969 [1961]: 25) diesen Gedanken auf kulturelle Erfahrungsstrukturen. Im ersten Teil von Wahnsinn und Gesellschaft behauptete er nach nur dürren Kommentaren zur Medizin der Antike, das Tragische sei „unweigerlich mit allen größeren Erfahrungen der Renaissance verbunden“ gewesen. Auch der Wahnsinn erscheine in der Malerei des 15. Jahrhundert noch als „Nichts“, Tod und „Wahnsinn der Welt“ (ebd.: 35, 43, 48, 65). Für den damaligen Menschen hätten „die Freiheit, selbst wenn sie erschreckend ist, seiner Träume und die Phantasmen seines Wahnsinns mehr Anziehungskraft als die begehrenswerte Realität des Fleisches“ gehabt (ebd.: 39).

Der „vorklassische“ Wahnsinn habe also noch auf die "tragische“ Verletzlichkeit und Reversibilität der Vernunft hingewiesen (ebd.: 111). Das Tragische wurde so für Foucault unter Nietzsches Einfluss zum Sublimat des vorprädikativen Seins und zum Mittler von Wahrheit (vgl. Wellbery 2007; Geisenhanslüke 1997: 195-212; Foucault 2006 [1961]: 237). Nur eine solche „vertikale Interpretation“ gestatte die Einsicht, dass der Wahnsinn auch heute noch „unter jeder seiner Formen auf eine vollständigere, gefährlichere Art auch diese tragische Erfahrung verbirgt" (Foucault 1969 [1961]: $49 \mathrm{f}$.$) .$

Dieses Motiv verankerte Foucault (ebd.: 66) insbesondere am historischen Material der um 1500 in ganz Europa beliebten Narrenliteratur (Sebastian Brant, Erasmus von Rotterdam). Die historische Richtigkeit dieser These haben Hans Christian Erik Midelfort (1999: 228-276) und andere Historiker seit den achtziger Jahren wiederholt bezweifelt (vgl. Jones \& Porter 1994; Scull 2007). Auch uns scheint Foucaults Identifikation von Narrheit und Wahnsinn falsch zu sein. Denn im Begriff der Narrheit erstarkte eine moralkritische Strömung in der Frühen Neuzeit, die menschliche Laster gegenüber der älteren theologischen Ethik in den Kreis der neuzeitlichen Individualitätsformen zog. Weder die Narren, noch die „nobilitierte“ Melancholietheorie der Renaissance, die Foucault weitgehend ignorierte, und auch nicht die dämonologisch verfemten „Hexen“ und „Zauberer“ galten im 16. Jahrhundert als Protagonisten des Wahnsinns. Dessen Phänomene wurden vielmehr im traditionellen Rahmen der hippokratisch-galenischen Fakultätenlehre des Geistes verhandelt (Brückner 2007: 209-215, 236-240). ${ }^{12}$

Im 17. Jahrhundert, so Foucault, sei dann die tragische Qualität des Wahnsinns durch ein neues und „kritisches“ gesellschaftliches Bewusstsein verdrängt worden, dessen Erfolg Foucault als Konsequenz des Werks 
von René Descartes darstellte. Descartes habe in den Meditationes von 1641 jede Option des vernünftigen Subjekts abgelehnt, sich in der Selbstvergewisserung auf eine Erfahrung des Wahnsinns berufen zu können. ${ }^{13}$ Die cartesische Wende sei somit das epistemologische Fanal der folgenden sozialen Exklusion: Mit der Gründung des Pariser Hôpital général im Jahr 1657 setze bis gegen Ende des 18. Jahrhunderts die „große Gefangenschaft" der Irren ein. Die moralische und institutionelle Gleichbehandlung von Verbrechen und Wahnsinn im Asyl ergebe sich dabei nicht aus der zunehmenden Medikalisierung des Wahnsinns, sondern die Ärzte fungierten „als Wächter, um die anderen vor der konfusen Gefahr zu schützen, die durch die Mauern der Internierung schwitzte." (ebd.: 363, Hervorhebung im Original, vgl. 68-98).

Auch diese These ist vielfach bezweifelt worden (vgl. Fauvel 2015; Scull 2007; Gordon 2007). Uns hingegen scheint zumindest ihre Stoßrichtung plausibel zu sein. Seit dem späten 17. Jahrhundert wurden in Europa hunderte „houses of correction“, „hôpitaux généraux“ oder „Zucht- und Tollhäuser" eingerichtet, in denen Kriminelle, Arme und Waisen, aber eben auch Irre, untergebracht wurden. ${ }^{14}$ Zweifellos handelt es sich um ordnungspolitische Strategien zur Abwehr von Armut im Rahmen des Absolutismus. Foucault unterschied zwar zwischen Frankreich und Großbritannien, aber unterschätzte wohl die gesamteuropäische Diversität und Ungleichzeitigkeit der Prozesse ebenso wie die öffentliche, in England auch von Insassen geäußerte, Kritik (vgl. dazu Brückner 2007: 306-345, 408-416). Seine Argumentation verband somit den Rekurs auf Nietzsche und die Kritik des cartesischen cogito mit der Institutionskritik von 1954, aber in klarer Distanz zum Marxismus und bereits mit Zügen einer Theorie der Disziplinarmacht. Im nächsten Schritt verknüpfte er seine Analyse der spezifischen Qualität des Wahnsinns in der französischen Klassik mit diesem kritischen Anspruch.

\section{Der Wahnsinn des Wahnsinns}

Im zweiten Teil von Wahnsinn und Gesellschaft bezog Foucault die gesellschaftliche Dynamik auf den Begriff des Deliriums und die damaligen Klassifikationssysteme (mit Einheiten wie Demenz, Manie und Melancholie, Hypochondrie). Historisch treffend erklärte Foucault (1969 [1961]: 196): „Die verwirrte und abwegige Vorstellungskraft, auf halbem Wege zwischen Irrtum und Versehen einerseits und körperlichen Störungen andererseits, ist nach übereinstimmender Benennung der Ärzte und Philosophen der klassischen Epoche als Delirium bezeichnet worden." In Beispielen aus zeitgenössischen Lehrbüchern fand er eine doppelte Struktur: Oberflächlich handele es sich um fehlerhafte Sprechakte (etwa die Aussage „Der, der und der sind meine Feinde; nun sind aber alle Männer; folglich sind alle 
Männer meine Feinde."). Untergründig fundiere solche Narrative jedoch ein organisierendes Prinzip, ein omnipräsentes und sprachlich manifestes „zweites“ Delirium als „erste und letzte Struktur des Wahnsinns“: „Das Delirium als Prinzip des Wahnsinns ist ein System falscher Sätze in der allgemeinen Syntax des Traumes.“ (ebd.: 245 f.). Der Wahnsinn äußere sich in der Umnachtung (,idios kosmos“) als Irrtum in der Grammatik eines Traums, dem Foucault wie schon 1954 einen irreduziblen Ausdruckswert zusprach. Die positive „Fülle“ von rational verknüpften Bildern im Wahnsinn manifestiere sich in der Logik einer quasi-vernünftigen Sprache, die zugleich irrational, ,abwesend“ und fremd bleibe:

Der Wahnsinn ist durch die Verbindung von Vision und Verblendung, Bild und Urteil, Phantasma und Sprache, Schlaf und Wachen, Tag und Nacht im Grunde nichts, denn er verbindet in ihnen, was sie an Negativem haben. Aber das Paradoxe dieses Nichts ist es, in Zeichen, Worten und Gesten dies zu manifestieren und aufbrechen $\mathrm{zu}$ lassen. (ebd.: 246, Hervorhebung im Original).

Damit aber sei jene fundierende, den Wahnsinn des Wahnsinns stiftende Struktur „gleichzeitig selbst Wahnsinn und jenseits jedes seiner Phänomene schweigende Transzendenz, die ihn in seiner Wahrheit konstituiert." Im Verhältnis zu diesem fundamental „abwesenden“ Wahnsinn habe die utilitaristische Vernunft der Aufklärung die konkreten Manifestationen des Wahns nur als ihr Gegenteil, als "geblendete Vernunft" begriffen (ebd.: 240). Die primäre Unvernunft, der Wahnsinn selbst, verschwinde hinter den Denunziationen der binären Vernunft genauso wie die Armen und Irren in der Internierung. Diese Architektur jener transzendenten Grundstruktur konkretisierte nicht nur Foucaults epistemische Prämissen, sondern verklammerte die sozialgeschichtliche Analyse mit der Logik des Gegenstands. Die hegemoniale Vernunft lösche demnach die Unvernunft im Wahnsinn und beraube ihn so seiner Quelle und Vitalität.

\section{Die Genese der Anstaltspsychiatrie}

Der dritte Teil von Wahnsinn und Gesellschaft rekonstruierte die Herausbildung der klinischen Psychiatrie im späten 18. und frühen 19. Jahrhundert. Die von England um 1750 ausgehenden Reformen des Armen- und Tollhauswesens sind historisch gut belegt (ärztliche Betreuung, staatliche Regulierung; vgl. Scull 2015: 137-161; Porter 1987; Dörner 1984 [1969]). Der aufklärerische Anspruch, die (heilbaren) Insassen als bürgerliche Subjekte $\mathrm{zu}$ rehabilitieren, wurde mit sensualistisch begründeten Milieutherapien umgesetzt. Foucault wertete dies aber keineswegs als medizinischen Fortschritt. Vielmehr seien ab 1760, nach einer französischen Handelskrise, neue Armen- und Arbeitshäuser eingerichtet worden. Die Gruppe der „ar- 
men Kranken" sei kaum in der Produktion einsetzbar gewesen und dann um 1780 im Namen humanitärer Ideale aus den Gefängnissen und Tollhäusern geholt worden. Foucault (1969 [1961]: 408-461) bestritt die fachliche Legitimation dieser „Befreiung“: Die Medizin habe lediglich juristische und politische Vorgaben akzeptiert, um die "Geisteskrankheiten“ als moralische Fehlentwicklungen festzuschreiben. Paradigmatisch sei Philippe Pinels (1745-1826) Sammelbegriff der „aliénation“ („Entfremdung“; Pinel 1801 [1800]: XIX f.). In dieser Störungskategorie, die Pinel keineswegs rein organisch begründete, sondern als Folge verselbständigter Affekte fasste, flossen laut Foucault (1969 [1961]: 127 f., 380-386) die „Begrenzungen der Subjektivität" (Krankheit) mit der strukturellen Exklusion (Internierung, Unmündigkeit) in individualisierter Form zusammen. So erklärte er nun, anders als 1954, den Begriff der „Entfremdung“, der hier begriffsgeschichtlich noch vor Hegel zu sehen ist, zu einem Teil des geschichtlichen Problems.

In den berühmten Reformanstalten von William Tuke (1732-1822; Yorker "Retreat") oder von Pinel in Paris seien also nach Foucault (ebd.: 479, 500-536) um 1800 emanzipative Begriffe der naturhaften Freiheit des Menschen durch die Kategorien des pathogenen oder aber heilsamen Milieus ersetzt worden. Der Wahnsinn habe nicht mehr als Gegenteil der Vernunft gegolten, sondern nur als „Widerspruch in der noch vorhandenen Vernunft", wie es später bei Hegel (1992 [1830]: 414) hieß. Die tragische Qualität des Wahnsinns sei zur individualmoralischen Psychologie der Schuld und Sozialisation depotenziert worden (Foucault 1969 [1961]: 406-416, 479). Darin liege das konkrete, historische Apriori der positivistischen Psychiatrie: „Es handelt sich nicht um eine Befreiung der Irren am Ende des achtzehnten Jahrhunderts, sondern um eine Objektivierung des Begriffs ihrer Freiheit." (ebd.: 542, Hervorhebung im Original; vgl. ebd.: 407, 459 f.). Die Psychopathologie des frühen 19. Jahrhunderts habe zwar einen neuen Zugang zum Wahnsinn eröffnet, doch ihre anthropozentrische Form verfehle dessen vitalen Kern:

Dadurch, daß er seinen Wahnsinn gemeistert hat, ihn in den Kerkern seines Blicks und seiner Moral gefangen hat, indem er ihn befreite, dadurch, daß er ihn entwaffnet hat, indem er ihn in eine Ecke seiner selbst zurückdrängte, war es dem Menschen möglich, schließlich jene Beziehung von sich selbst, zu sich selbst herzustellen, die man ,Psychologie‘ nennt. (ebd.: 15).

Die Kategorie des individuellen Selbstbewusstseins, die für Foucault 1954 noch die Freiheit der Existenz schützte, galt nun als ein Teil der Bedingungen der Möglichkeit, die "Geisteskrankheit" als humanwissenschaftlichen Gegenstand zu umgrenzen. 
Prämissen, Aporien und Weiterentwicklung

Wahnsinn und Gesellschaft enthielt deutliche Spuren der früheren Arbeiten Foucaults, insbesondere den Gedanken einer autonomen, für den Diskurs und das Bewusstsein opaken Qualität des Wahnsinns im Sinne einer Freiheit, die sich „nur von der Höhe der Festung her [versteht], die ihn gefangen hält" (ebd.: 13). Diese Qualität fasste er wie bereits 1954 als idiosynkratischen Kosmos mit tragischer Wahrheit. Ebenso erhalten blieb die sozialhistorische Kritik der Objektivierungsprozesse. Diese Spuren verwischte Foucault 1961 im Rekurs auf Nietzsche, Lacan und Roussel. Doch frische Referenzen erzeugten neue Widersprüche: Die Hypothesen zur primären Trennung zwischen Sinn und Nicht-Sinn und zur tragischen Qualität der Unvernunft umgingen das methodologische Problem des historischen Objekts, sofern dieses nun in schweigender "Transzendenz“ wirke (Foucault 1969 [1961]: 240). Die Idee von der „Abwesenheit des Wahnsinns“ blieb abstrakt - ohne Verankerung in der geschichtlichen Wirklichkeit der Subjekte des Wahnsinns selbst. In der Konsequenz erklärte Foucault das vorcartesische Bewusstsein vom Wahnsinn zum „Punkt Null“ von dessen Geschichte in der Moderne fast ohne Rekurs auf die Antike, deutete die Narrheit zum Wahnsinn um und übersah die Bedeutung der Melancholie und Dämonologie. Fragwürdig bleiben auch die Thesen zur "großen Gefangenschaft" der Irren oder aber zur Genese der Anstaltspsychiatrie am Maßstab der Entfremdungstheorie ohne Bezug auf weitere Strömungen der Spätaufklärung (vgl. dazu Brückner 2007: 301-472).

Die Folgen der 1961 eingeführten epistemischen Prämissen waren gravierend. Lesen wir Foucaults Argumentation als implizite Auseinandersetzung mit Immanuel Kant, versuchte er, wie Béatrice Han (2002) gezeigt hat, die Bedingungen der Möglichkeit der Erkenntnis des Wahnsinns aufzudecken und blieb insofern erkenntniskritisch. Foucault beabsichtigte aber keine transzendentalphilosophische Kritik, sondern versuchte, das „historische Apriori“ der modernen Rede von den Geisteskrankheiten freizulegen. So konnte ihm Jürgen Habermas (1985: 296, 282) „gnadenlosen Historismus" und ein kryptoromantisches Verhältnis zu seinem Gegenstand vorwerfen. In der Tat räumte Foucault (1981 [1969]: 29) später ein, er habe 1961 mit dem Begriff der Erfahrung noch „ein anonymes und allgemeines Subjekt der Geschichte“ zugestanden. Wahnsinn und Gesellschaft zeigte Foucault also erst auf dem Weg zur späteren Diskursanalyse und Analytik der Macht. Ebenso evident ist die philosophische Originalität und die kritische Wirkung des Werks, sein Materialreichtum und poetischer Glanz. Foucaults Idee, die Sprache des Wahnsinns als Schweigen zu würdigen, öffnete der Sache selbst einen neuen, abgründigen Raum des Seins. 
In den folgenden zwölf Jahren publizierte Foucault einige kleinere Texte zum Thema (etwa 2001 [1962a], 2001 [1964], 2002 [1972]). Bereits 1963 erschienen parallel die literaturtheoretische Monographie über Roussel und die medizinhistorische Studie Die Geburt der Klinik. Dort analysierte Foucault (1988 [1963]: 13) die Rationalität, Struktur und Empirie der Medizin des 19. Jahrhunderts im Kontext ihrer historisch konstitutiven Bedingungen und der kulturellen Bedeutung des Todes. Die Befunde arbeitete er 1966 in Die Ordnung der Dinge zum Konzept der „Episteme“ aus, die das Wissen einer Epoche in verschiedenen Disziplinen strukturell charakterisieren. Demnach gehöre auch die Phänomenologie zum anthropologischen Epistem der mit Kant beginnenden Moderne (Foucault 1974 [1966]: 367-413).

In der Archäologie des Wissens von 1969 präzisierte er die Methodik der Diskursanalyse. Sie beschreibe nicht mehr wissenschaftliche Gegenstände, wie etwa in Wahnsinn und Gesellschaft, sondern allein die sprachlichen Praktiken „ohne Beziehung zum Grund der Dinge“ (Foucault 1981 [1969]: 72). Den Übergang zur Theorie der Macht markierte die Inauguralvorlesung am Collège de France von 1970. Dort konstatierte Foucault (1977 [1971]: 13): „Drei große Ausschließungssysteme treffen den Diskurs: das verbotene Wort; die Ausgrenzung des Wahnsinns; der Wille zur Wahrheit." Im Rahmen der Diskursanalyse, die sich den Prinzipien der „Umkehrung“, „Diskontinuität“, „Spezifität“ und „Äußerlichkeit“ bediene, um das serielle „Ensemble diskursiver Ereignisse“ zu erkennen, wurden die Themen „Wahnsinn“ und „Psychiatrie“ systematisch verortet. Im binären Verhältnis zwischen Vernunft und Wahnsinn zeige sich das Prinzip des Ausschlusses und um die Genese der (institutionellen) Kontrolle des Diskurses zu beschreiben, eigneten sich "Kritik“ und „Genealogie“ (ebd.: $42 \mathrm{f}$.). Tatsächlich wandte Foucault sich dem Thema in der Vorlesung zum Wintersemester 1973/74 unter dem Titel Die Macht der Psychiatrie (Le pouvoir psychiatrique) nochmals zu.

\section{Die Produktivität des Wahnsinns}

Die Vorlesungen von 1973/74 zur französischen Psychiatrie des 19. Jahrhunderts sind bislang wenig rezipiert worden, obwohl sie zeitlich und inhaltlich an Wahnsinn und Gesellschaft anschließen. ${ }^{15}$ Laut Foucault (2005 [2003]: 28-34, 2003 [1976]) hätten 1961 noch Begriffe der „Gewalt“ oder „Institution“ im Mittelpunkt gestanden, nun aber gehe es um das „Dispositiv der Macht als Erzeugerinstanz der diskursiven Praxis“ (Foucault 2005 [2003]: 29). Macht sei keine individuelle Fähigkeit (wie etwa bei 
Max Weber), sondern eine distributive, relationale Praktik (Foucault 1983 [1976]: 113-138), wie er 1976 im ersten Band von Sexualität und Wahrheit betonte. Im Kontext der Psychiatrie wirke Macht nicht nur repressiv, sondern auch produktiv in einem ständig neu konstellierten "Anstaltskampf“ zwischen Ärzten und Insassen (Foucault 2005 [2003]: 495). Die übergreifende, bis heute gültige Machtstruktur sei die "Disziplinarmacht" (ebd.: 67-94). Ihr zentrales Prinzip, die Umformung individuellen Verhaltens anhand präskriptiver Normen, erhob Foucault 1975 zum Titel seines Buches Überwachen und Strafen. Ihre historischen Ursprünge lägen in den politisch-ökonomischen Anforderungen und den militärischen, pädagogischen und polizeilichen Disziplinartechniken des 16. und 17. Jahrhunderts (ebd.: 81-84, 1977 [1975]: 173-220). Paradigmatisch für ihre invasive Qualität sei die Praxis des panoptischen Blicks in Jeremy Benthams (1748-1832) Gefängnisarchitektur. Die kreisförmige Anordnung der Zellen um einen zentralen Turm erlaube den Aufsehern, zu beobachten ohne selbst sichtbar zu sein und zwinge die Insassen zur ständigen Selbstkontrolle (Foucault 2005 [2003]: 117). Methodisch würden die jeweiligen „Macht/WissensKomplexe“ im Rückgriff auf Nietzsche (1999 [1887]: 253) durch eine „genealogische" Rekonstruktion der inneren Logik in der Genese historischer Sachverhalte aufgewiesen (Foucault 2005 [2003]: 99-140).

\section{Das psychiatrische Dispositiv des 19. Jahrhunderts}

Um die faktisch existierenden Strukturen der Disziplinarmacht als „Dispositiv" zu erfassen, analysierte Foucault typische Praktiken und Szenen aus den Lehrbüchern der Zeit. Eine „Protoszene der Geschichte der Psychiatrie" sei Pinels (1801 [1800]: 205-206, vgl. 305-309) Referat der Behandlung des englischen Königs George III. (1738-1820), der ab 1765 mehrfach an erheblichen psychischen Verstörungen litt (vgl. Peters \& Beveridge 2010). Pinel beschrieb eine Szene, in der eine schwere Krise des Königs, der 1789 durch eine Regency Bill entmachtet werden sollte, mit unmittelbarer Gewalt behandelt wurde. Für Foucault (2005 [2003]: 40-62) signalisierte dies den Wandel von der absolutistischen Souveränität zur modernen Disziplinarmacht. Die psychiatrische Macht werde durch "Taktiken“ der "mentalen Orthopädie" erzeugt (ebd.: 58), insbesondere in der ärztlichen Visite (ebd.: 401) und der Lehrdemonstration von Fällen (ebd.: 402). Das Anstaltsmilieu wirke wie ein omnipräsenter panoptischer Blick und verdoppele die Sichtweise der Ärzte („Tautologie der Anstalt“, ebd.: 252).

Foucault griff dabei zu einem - wie er selbst zweifelnd feststellte "pseudomilitärischen Vokabular" der "Taktiken“ und „Strategien“ (ebd.: 34). Die auf Nietzsches Begriff der Kraft (vgl. Deleuze 1987: 112) beruhende Machtanalytik setzte somit die frühere Institutionenkritik fort: Ohne „Makrophysik“ gebe es keine „Mikrophysik der Macht“ (vgl. Lemke 2011: 
120-126). Handelnde Individuen kamen nur als Konstrukt und „Effekt der Macht“ vor, sofern „die Macht ein Verfahren der Individualisierung“ sei (Foucault 2005 [2003]: 32). Angesichts der gesellschaftlichen Praktiken, die den Wahnsinn in seinen konkreten historischen Formen provozieren und definieren, trat die noch 1961 im Kontext des Begriffs der Erfahrung so virulente Frage nach dem Gegenstand der Untersuchung in den Hintergrund. Nun zählten auch die Sprache, Diskurse und Formen des Wissens zu den Machtpraktiken. Zur philosophischen Begründung berief sich Foucault auf den späten Nietzsche und dessen perspektivistische Kräftemetaphysik des „Willens zur Macht“ (vgl. Riccardi 2014). Diese Position stellte er nie mehr grundsätzlich in Frage. Welchen systematischen Ort und welche analytische Kraft besaß aber dann die von ihm verwendete Kategorie der „Geisteskrankheit"?

\section{Wissen und Normierung}

Foucault behandelte die Rede von den „Geisteskrankheiten“ als historisch konkrete Erscheinungsform eines bestimmten humanwissenschaftlichen Wissens im Kontext von Machtbeziehungen. Die Kategorie der „Geisteskrankheit" entstehe als Form des Wissens im Zuge einer tiefgreifenden Normierung der Individuen in der psychiatrischen Praxis. In der Psychiatrie des 19. Jahrhunderts sei das Wissen über die Geisteskrankheiten durch Befragungen, die Verwendung von Drogen oder Hypnose gewaltsam produziert worden (Foucault 2005 [2003]: 417). Die Befragung stehe - anders als die rein medizinische Anamnese - in der historischen Kontinuität der Beichte und des Geständnisses (ebd.: 397 f.). Sie bestätige den Wahnsinn als Krankheit und den Psychiater als Experten: „Gib mir deine Symptome, mach mir aus deinem Leben Symptome, und du wirst aus mir einen Arzt machen“ (ebd.: 401). Auch seien durch die professionelle Hypnose bei „Hysterikern“ essentielle „Krankheitsbeweise“ erzeugt worden (ebd.: 417) wie umgekehrt Patienten, die hypnotische Effekte simulierten, die „wahren Aktivisten der Antipsychiatrie“ gewesen seien (ebd.: 366).

Das psychiatrische Dispositiv gehe auch von den Familien aus (ebd.: 394). Diese hätten ein vitales Interesse, ihre Angehörigen aus den Anstalten für die Erwerbstätigkeit zurückzugewinnen („Anomalieprofit“ der Psychiatrie, ebd.: 182). Zudem beruhe das Dispositiv auf einer „Psychologisierung des Kindes“, speziell durch die Psychoanalyse, aber auch mittels der Kategorie der „Anormalität“, die anhand der Typen von „Idiotie, Schwachsinnigkeit, Blödheit“ definiert worden sei (ebd.: 295). Ursprünglich habe der "Idiot“ als eine „Art von Kind“ und nicht als krank gegolten, aber dennoch nie der gesunden Norm entsprechen können. Dieses normative Prinzip präge die gesamte Psychopathologie (ebd.: 302, 319). 
Folgerichtig hieß die folgende Vorlesung am Collège de France Die Anormalen. Foucault (2007 [1999]: 405, 2001a [1977]) untersuchte nun, wie "das Verhalten und seine Abweichungen und Anomalien“ im ausgehenden 19. Jahrhundert zum Gegenstand von Psychiatrie, Psychologie oder Kriminologie wurden, indem das psychiatrische Dispositiv um die forensische Kategorie des „gefährlichen Menschen“ erweitert worden sei. Dieses Thema bestimmte auch den von Foucault 1973 herausgegebenen Sammelband über den Mörder Pierre Rivière (1815-1840).

\section{Gegenwartsanalyse und Antipsychiatrie}

Die Vorlesungen von 1973/74 dokumentieren eine gegenwartsanalytisch neu justierte Position. Aber so klar die Konturen des theoretischen Anliegens auch hervortreten, so selektiv wirken manche Analysen. So klammerte die Behauptung, der normative Gehalt des psychiatrischen Krankheitsbegriffs lasse sich auf Bestimmungen der „Idiotie“ zurückführen, bedeutsame zeitgenössische Kontroversen aus (etwa zur Naturphilosophie und Nervenphysiologie; vgl. Brückner 2011). Überhöht erscheint auch Foucaults (2005 [2003]: 408) Bewertung von Jacques-Joseph Moreaus (1845) Cannabis-Experimenten, die ein „völlig neues Verstehen“ ermöglicht hätten. Vor allem aber vernachlässigte er die so immens folgenreiche französische Degenerationslehre (Bénédict A. Morel; Valentin Magnan). Auf ein weiteres Problem hat Chris Philo (2007: 162) hingewiesen: Foucaults tendenzielle Heroisierung der Hysterikerinnen. Dies hängt offenbar mit einem problematischen Begriff der „Antipsychiatrie“ zusammen, den Foucault verwendete und der einer Kontextualisierung bedarf.

Laut Foucault bekämpfe die „Antipsychiatrie“ die Psychiatrie von innen, um „dem Kranken selbst die Macht zu geben, seinen Wahnsinn und die Wahrheit seines Wahnsinns zu produzieren“ (2005 [2003]: 501-504). Trotz Gemeinsamkeiten mit der Psychiatriekritik seiner Zeit (Franco Basalgia, David Cooper, Erving Goffman, Ronald D. Laing, Thomas Szasz) zielten solche Äußerungen nicht nur auf die repressive Funktion der Institution. Vielmehr umfasste Foucaults allzu summarischer Begriff der „Antipsychiatrie“ auch die "Gegenmacht“ der Einzelnen, ihren „Wahnsinn bis an sein Ende zu führen“, um die „Umdeutung des Wahnsinns zur Geisteskrankheit rückgängig zu machen“ (ebd.: 504). ${ }^{16}$ Hier hielt Foucault an dem Gedanken einer vom Diskurs unabhängigen (subversiven) Dynamik fest, doch nun ersetzten gewöhnliche Patienten die in Wahnsinn und Gesellschaft präsentierten literarischen Kronzeugen.

Damit schließt sich der Kreis zur Rezeption seines Werks. Foucault galt in den fünfziger Jahren als phänomenologisch orientierter Psychologe. Und Wahnsinn und Gesellschaft wurde im sozialpsychiatrischen Kontext durchaus als Kritik kustodialer Strukturen begrüßt (vgl. Basso 2015; Eribon 1999 
[1989]: 197-201; Leibbrand 1964). Dies änderte sich Ende der sechziger Jahre mit den 1965 und 1967 publizierten, englischsprachigen Übersetzungen des Buchs. Colin Gordon (1990) hat diese stark gekürzten (und 1967 von Cooper eingeleiteten) Ausgaben für gravierende Fehlrezeptionen und die in den achtziger Jahren einsetzende Kritik an Foucaults historischer Empirie verantwortlich gemacht (vgl. Still \& Velody 1992; Scull 2007). Zudem trug Foucaults Politisierung nach dem Pariser Mai 1968 zur Distanzierung reformorientierter Ärzte bei. Der Hannoveraner Klinikdirektor Karl Peter Kisker (1979: 821) berichtete etwa, der führende französische Psychiater Henri Ey (1900-1977) habe Wahnsinn und Gesellschaft als das „große Rotbuch der Antipsychiatrie“ bezeichnet (vgl. Delille 2008: 315-319). Und Klaus Dörner (1984 [1969]: 336) schätzte zwar Foucaults Wissenssoziologie, warf ihm aber Einseitigkeit und Praxisferne vor. Im Kontext dieser Kontroversen führte Foucaults zeitkritische Haltung offenbar auch zur tendenziellen Identifikation von Hysterie und subjektivem Widerstand in den Vorlesungen von 1973/74.

Halten wir fest, dass Foucault zuletzt den Wahnsinn der Patienten weder als gegebene Erfahrung (wie 1954) noch als „abwesend“ (wie 1961) bestimmte, sondern versuchte, die sozialen Operationen zu beschreiben, durch die er als Krankheit erkannt werde (2005 [2003]: 215, 387). Die Psychiatrie behaupte das „absolute Recht des Nicht-Wahnsinns über den Wahnsinn“ und disqualifiziere „das von dieser Krankheit befallene ,Subjekt“" zu einem Menschen, „der keinerlei Macht und keinerlei Wissen über seine Krankheit“ besitze (ebd.: 503). Die Kategorien zielten somit wesentlich direkter und körperlicher als in Wahnsinn und Gesellschaft auf die konkrete Praxis der Psychiatrie. Doch von der einst angenommenen „Tragik“ der „Unvernunft“ war keine Rede mehr. Vielmehr sollten schlichtweg deskriptiv erfasste „Krankheiten“ als widerständiges Potenzial erkennbar werden. Das Problem des historischen Objekts, das Foucault in Wahnsinn und Gesellschaft zu umgehen versuchte, verschwand scheinbar im Blick auf die Praktiken, mit denen wissenschaftliche Gegenstände als solche erst konstituiert werden.

\section{Foucaults philosophische Historiographie des Wahnsinns}

Es gibt wohl kaum einen Philosophen des zwanzigsten Jahrhunderts, für den die Themen "Wahnsinn und Psychiatrie" so relevant waren wie für Michel Foucault. Der frühe Foucault sah „Geisteskrankheiten“ 1954 als phänomenologisch „positiv“ ausweisbares Phänomen der subjektiven Erfahrung, die quer zur „normalen“ Erfahrungswelt stehe. In Wahnsinn und 
Gesellschaft stellte er hingegen die Unvernunft als Urgrund des Wahnsinns und kulturell „abwesendes“, nur „negativ“ fassbares Phänomen dar. In Die Macht der Psychiatrie verteidigte er das Konzept einer verborgenen Wahrheit des Wahnsinns, der in der Praxis des „Anstaltskampfs“ als „Krankheit“ eminent produktiv wirke. In dieser Linie formierte sich sein Denken jeweils als Vorbereitung folgender Arbeiten. Die frühe Kritik des Strukturalismus mit der Idee einer sich der Logik des Diskurses entziehenden Struktur der Erfahrung führte zur These der „Abwesenheit“ des Wahnsinns und das Konzept der siebziger Jahre erhielt deren subversive Momente. In den achtziger Jahren sehen wir dann einen kritisch argumentierenden Foucault (2005 [1984]: 848), der sich ohne transzendentalphilosophisches Anliegen den Prinzipien der Aufklärung im Sinne einer historischen „Ontologie unserer selbst“ verpflichtet sah. Foucault bestand auf dem Primat der geschichtlichen Kritik. Ihre Entwicklung lässt sich am Themenkomplex „Wahnsinn und Psychiatrie“ durchgehend zeigen.

Foucaults ungebrochene Attraktivität für die heutigen Geistes- und Sozialwissenschaften (Kammler et al. 2008; Buchmann \& Cserni 2008) steht in deutlichem Kontrast zu seiner kontroversen Rezeption in der Psychiatriegeschichtsschreibung (Dinges 1994) und vor allem zu seiner geringen Wirkung auf die psychiatrische Praxis. Wir sehen nur einige Arbeiten über Disziplinarstrukturen in der Gemeindepsychiatrie (Iliopoulos 2012: $53 \mathrm{f}$;; Bracken \& Thomas 2010), biopolitische Strategien (Gahr \& Cabanis 2014; Kollek \& Lemke 2008) oder zur Ökonomisierung von Diagnostik und Versorgung (Leoni 2013: 93 f.; vgl. Heinze 2008). In der Psychiatriegeschichtsschreibung ist eine Zurückhaltung spürbar, die sich aus der Skepsis zum Verhältnis von historischer Empirie und Theorie bei Foucault ergeben hat (vgl. Scull 2007; Gordon 2007). Vor allem in Frankreich wird kontrovers diskutiert. Dort steht die Anziehungskraft von Foucaults Psychiatriekritik möglicherweise auch im Kontext der verspäteten, nicht vor den neunziger Jahren erfolgten Aufarbeitung der französischen Psychiatrie im Zweiten Weltkrieg (vgl. von Bueltzingsloewen 2007), wobei Positionen, die Foucault kritisch sehen, teils heftig angegriffen werden (Bacopoulos-Viau \& Fauvel 2016: 10). In der neueren deutschsprachigen Psychiatriegeschichtsschreibung wird Foucaults Werk mittlerweile durchaus eingebunden (vgl. Schmiedebach 2016: 2 f.; Wernli 2014: 18, 32; Balz 2010: 182 ff.).

So gibt es durchaus Schnittstellen seines Ansatzes zur neueren Sozialund Patientengeschichte der Psychiatrie. Foucault (2005 [2003]: 230 f.) gestand zwar in den Vorlesungen ein, er wisse kaum etwas über Selbstberichte von Insassen und lehnte es 1976 ab, „eine Geschichte der Wahnsinnigen zu schreiben" (Foucault 2003 [1976]: 121), nutzte aber nach 1961 mehrfach selbst autobiographische Dokumente (Foucault 2001 [1962a], 1989 [1963], 1975 [1973], 2001a [1977]). Paradoxerweise könnten seine einst vom Stand- 
punkt der Fortschrittsgeschichte als „revisionistisch“ erscheinenden Positionen selbst revidiert werden, sofern patientengeschichtliche Quellen nicht nur institutionelle Zwänge, sondern auch die kritische Kultur und öffentliche Aktivität von Insassen belegen (vgl. Bacopoulos-Viau \& Fauvel 2016; Fauvel 2015; Brückner 2007) - was wiederum an die Analytik der Macht anschließbar wäre.

Der zentrale Gewinn der Beschäftigung mit Foucault liegt unserer Ansicht nach in der Sensibilisierung für die Nachteile ideengeschichtlicher und empiristischer Ansätze sowie für die Vorteile der philosophischen Reflexion des psychiatrischen Feldes (vgl. Fulford et al. 2013). In einem doppelten Sinn hat Foucault gezeigt, wie eng historische Empirie mit theoretischer Reflexion zusammenhängt, nämlich mit seiner Kritik der traditionellen Methodologie, aber implizit ebenso angesichts seiner eigenen, teils problematischen epistemischen Prämissen. Foucaults Arbeiten betonen somit die Spielräume zwischen geschichtswissenschaftlicher Erfahrung und philosophischem Denken. Er bot Modelle der Reflexion an, um die Dinge zu Ende zu denken, kritische Modelle, die das Denken der Tatsachen herausfordern - darin liegt ihre Attraktivität.

Dies betrifft im Anschluss an Foucault etwa die Analysen von Ian Hacking (2004) oder Nikolas Rose (2006) zum artifiziellen Status psychiatrischer Klassifikationen oder Zygmunt Baumans (2000: $9 \mathrm{f} ., 70$ ) Überlegungen zum Wandel von panoptischen zu „synoptischen“ Formen sozialer Kontrolle, in denen das Bedürfnis gesehen $\mathrm{zu}$ werden zentral sei. Ebenso beschrieb Alain Ehrenberg (2015 [1998]) die Transformation der Disziplinargesellschaft in eine Leistungsgesellschaft, die Personen zu erschöpften Unternehmern ihrer selbst mache. Auch ließe sich die spätmoderne „Sympathie mit der Schizophrenie“ (Leferink 1997) mit Foucault als Ausdruck der Verschiebung psychiatrischer Kategorien in das Zentrum der sozialen Normalität verstehen. Der „Wahnsinn“ wäre dann aus Sicht der medical humanities, wie Angela Woods (2011) vorschlägt, nicht mehr subjektiver, negativer oder produktiver Gegenpart unserer Kultur, sondern ein der Struktur spätmoderner Gesellschaften ähnelndes Phänomen. 


\section{Anmerkungen}

1 Siehe zu neueren themenspezifischen Überblicken: Basso (2016b); Basso (2015); Leoni (2013); Hacking (2011); Heinze (2008); Cutting (2005). Zur psychiatriehistorischen Rezeption: Bacopoulos-Viau \& Fauvel (2016); Fauvel (2015); Dinges (1994); Jones \& Porter (1994); Still \& Velody (1992); zur Psychoanalyse: Hirsch (2010). Zur allgemeineren neueren Rezeption: Raffnsøe et al. (2015); Segre (2014: 79-112); sowie das Themenheft der Zeitschrift History of the Human Sciences (Koopman 2011). Zur deutschsprachigen Rezeption: Kammler et al. (2008); Buchmann \& Cserni (2008); Unterthurner (2007); Honneth \& Saar (2002).

2 Vgl. Basso (2016a); Bert \& Basso (2015); Joranger (2016); Basso (2012); Woods (2011: 17 f.); Gondek (2008); Novella (2008); May (2005); Rüb (1990); Macherey (1986). Die späte Anerkennung des Frühwerks erklärt sich durch die bis in die achtziger Jahre reichende Skepsis gegenüber der philosophischen Anthropologie in Frankreich, zu der auch Foucault selbst beitrug.

3 Siehe Thoma (2016) zum Begriff des Tragischen im damaligen historischen Kontext.

4 Wir zitieren aus der ersten französischen Ausgabe von 1954 auf Basis der deutschen Übersetzung (1968) der zweiten französischen Auflage von 1962, nur wenn letztere von der ersten Auflage abweicht, handelt es sich um unsere Übersetzungen. Vgl. auch Foucaults (2001 [1957a], 2001 [1957b]) Recherchen zur Geschichte der Psychologie.

5 Hier ist insbesondere an den Einfluss Canguilhems (1958) zu denken, der die epistemologischen Prämissen der Psychologie untersuchte und deren Expertise, „die Verhältnisse des Menschen zum Menschen zu managen“ (ebd.: 24, unsere Übers.), in Verbindung mit dem Polizeiwesen brachte.

6 Wir zitieren aus der gekürzten deutschen Übersetzung von 1969, darin nicht enthaltene Passagen werden mit der englischen Ausgabe von 2006 belegt. Zur jüngeren Rezeption siehe: Bert \& Basso (2015); Hacking (2011); Artières \& Bert (2011); Geisenhanslüke (2008); Heinze (2008); Unterthurner (2007); Cutting (2005); vgl. auch die wiederholten Debatten zwischen Andrew Scull (2007) und Colin Gordon (1990, 2007); sowie die Sammelbände von Jones \& Porter (1994); Still \& Velody (1992).

7 Retrospektive Diagnosen als Mittel der historischen Analyse verwarf Foucault (1969 [1961]: 212, 129, 2006 [1961]: 536 f.); vgl. dazu Derrida (1976 [1967]: 58), sowie generell Stolberg (2012).

8 Die Übersetzung von 1969 (Foucault 1969 [1961]: 11) „Fehlen einer Arbeit“ ist überholt, treffender ist „Fehlen eines Werkes“; vgl. z. B. Mazumdar (2008: 454).

9 Siehe zum Begriff des „Autors“ Foucault (1988 [1969]) und zur „Archäologie“ Ebeling (2000).

10 In Die Ordnung der Dinge läuft dieser Gedanke auf eine Kritik der Repräsentation hinaus (Foucault 1974 [1966]: 78 ff.). Zu Foucaults Begriff der Erfahrung vgl. Unterthurner (2007).

11 Foucault (1969 [1961]: 235, 411) erwähnte lediglich Bernhard Bluet d'Arbères (15661606) und Jean Antoine Thorin (ca. 1733-1802), dessen Texte er allerdings falsch datierte (vgl. Brückner 2007: 352-355), sowie Protestschriften des 18. Jahrhunderts.

12 Vgl. werkbiographisch Bert \& Basso (2015), sowie Kastens (1992: 248) Hinweis auf die doppelte Bedeutung des Worts "folie“ als "Wahnsinn" und "Narrheit“.

13 Jacques Derrida widersprach dem 1967 mit der Behauptung, das cartesische Subjekt überdauere auch im Wahnsinn als Cogito (vgl. James 2011).

14 Vgl. Sachße \& Tennstedt (1998: 85-132). Das Pariser Hôpital beherbergte nach wenigen Jahren ca. 6000 Arme. Um 1700 gab es in ganz Frankreich gut einhundert „hôpitaux généraux" und bis 1789 stieg ihre Zahl auf 167 mit ca. 60.000 Insassen (Brockliss \& Jones 1997: 681). 
15 Vgl. zum Kontext Lagrange (2005 [2003]); zur Rezeption Leoni (2013); Beljan (2008); Elden (2006).

16 Siehe zum Begriff der „Antipsychiatrie“ Barberi (2011); Dain (1994); zu Foucaults (2003 [1976]) Nähe zur Psychiatriekritik Bracken \& Thomas (2010); Heinze (2008: $421 \mathrm{f}$.), Lagrange (2005 [2003]: 521-528), Eribon (1999 [1989]: 195-201); sowie die problematische Rezeption Foucaults als „antipsychiatrisch“ bei Colucci (2006) oder Matthews (1995).

\section{Literatur}

Akavia, Naamah 2008. Writing „The case of Ellen West“: Clinical Knowledge and Historical Representation. Science in Context (21): 119-144.

Artières, Philippe und Jean-François Bert 2011. Un succès philosophique: L'Histoire de la folie à l'âge classique de Michel Foucault. Caen: Presses Universitaires de Caen.

Bacopoulos-Viau, Alexandra und Aude Fauvel 2016. The Patient's Turn. Roy Porter and Psychiatry's Tales, Thirty Years on. Medical History (60): 1-18.

Balz, Viola 2010. Zwischen Wirkung und Erfahrung - eine Geschichte der Psychopharmaka. Neuroleptika in der Bundesrepublik Deutschland, 1950-1980. Bielefeld: transcript.

Barberi, Alessandro 2011. „Der Wahnsinn ist eine soziale Konstruktion.“ Zur Aktualität der Antipsychiatrie. Internationale Zeitschrift für Sozialpsychologie und Gruppendynamik in Wirtschaft und Gesellschaft (36/2): 3-18.

Basso, Elisabetta 2012. On Historicity and Transcendentality Again. Foucault's Trajectory from Existential Psychiatry to Historical Epistemology. Foucault Studies (14): 154-178.

Basso, Elisabetta 2015. Histoire, philosophie et pratiques de la psychiatrie: l'impact de l'œuvre de Foucault en Italie et Allemagne. In: Pascal Hintermeyer (Hg.). Foucault post mortem en Europe. Strasbourg: Presses Universitaires de Strasbourg: 69-80.

Basso, Elisabetta 2016a. À propos d'un cours inédit de Michel Foucault sur l'analyse existentielle de Ludwig Binswanger (Lille 1953-54). Revue de synthèse (137): 35-59.

Basso, Elisabetta 2016b. Foucault entre psychanalyse et psychiatrie »Reprendre la folie au niveau de son langage». Archives de Philosophie (79): 27-54.

Bauman, Zygmunt 2000. Liquid Modernity. Cambridge: Polity Press.

Beljan, Magdalena 2008. Vorlesungen. In: Clemens Kammler, Rolf Parr und Ulrich-Johannes Schneider (Hg.). Foucault-Handbuch. Stuttgart: Metzler: 138-149.

Bert, Jean-François und Elisabetta Basso (Hg.) 2015. Foucault à Münsterlingen. À l'origine de l'Histoire de la folie de l'Histoire de la folie. Paris: Editions de l'Ecole des Hautes Etudes en Sciences Sociales.

Binswanger, Ludwig 1994 [1930]. Traum und Existenz. In: Ders. Ausgewählte Werke in vier Bänden. Bd. 3. Heidelberg: Asanger: 95-119.

Binswanger, Ludwig 1945. Der Fall Ellen West. Eine anthropologisch-klinische Studie. Zürich: Orell Füssli.

Bracken, Pat und Philip Thomas 2010. From Szasz to Foucault: On the Role of Critical Psychiatry. Philosophy, Psychiatry \& Psychology (17): 219-228.

Brockliss, Laurence und Colin Jones 1997. The Medical World of Early Modern France. Oxford: Clarendon.

Brückner, Burkhart 2007. Delirium und Wahn. Geschichte, Selbstzeugnisse und Theorien von der Antike bis 1900. Bd. 1: Vom Altertum bis zur Aufklärung. Hürtgenwald: Pressler.

Brückner, Burkhart 2011. Moral, Freiheit und Natur - Die Ursprünge der europäischen Psychiatrie im Schnittpunkt von Aufklärung und Romantik. Sozialpsychiatrische Informationen (41/3): 8-12.

Buchmann, Michael und Iris Cserni 2008. Die Rezeption der Texte Michel Foucaults. Eine Untersuchung anhand der deutschsprachigen Literaturwissenschaft und Soziologie. Saarbrücken: Müller.

Bueltzingsloewen, Isabelle von 2007. L'hecatombe des fous. La famine dans les hopitaux psychiatrique français sous l'Occupation. Paris: Aubier. 
Canguilhem, Georges 1958. Qu'est-ce que la psychologie? Revue de Métaphysique et de Morale (63): $12-25$.

Colucci, Mario 2006. Foucault and Psychiatric Power after Madness and Civilization. In: Alain Beaulieu und David Gabbard (Hg.). Michel Foucault and Power Today. Lanham: Lexington Books: 61-70.

Cutting, Gary 2005. Foucault and the History of Madness. In: Gary Cutting (Hg.). The Cambridge Companion to Foucault. 2. Aufl. Cambridge: Cambridge University Press: 49-73.

Dain, Norman 1994. Psychiatry and Antipsychiatry. In: Mark S. Micale and Roy Porter (Hg.). Discovering the History of Psychiatry. New York: Oxford University Press: 415-444.

Deleuze, Gilles 1987. Foucault. Frankfurt a. M.: Suhrkamp.

Delille, Emmanuel 2008. Réseaux savants et enjeux classificatoires dans le Traité de Psychiatrie de l'Encyclopédie Médico-Chirurgicale (1947-1977). L'exemple de la notion de psychose. Thèse de doctorat, EHESS Paris.

Derrida, Jaques 1976 [1967]. Cogito und die Geschichte des Wahnsinns. In: Ders. Die Schrift und die Differenz. Frankfurt a. M.: Suhrkamp: 53-101.

Dinges, Martin 1994. The Reception of Michel Foucault's Ideas on Social Discipline, Mental Asylums, Hospitals and the Medical Profession in German Historiography. In: Colin Jones und Roy Porter (Hg.). Reassessing Foucault: Power, Medicine and the Body. London/New York: Routledge: 181-212.

Dörner, Klaus 1984 [1969]. Bürger und Irre. Zur Sozialgeschichte und Wissenschaftssoziologie der Psychiatrie. Frankfurt a. M.: Fischer.

Dreyfus, Hubert L. und Paul Rabinow 1987 [1982]. Michel Foucault. Jenseits von Strukturalismus und Hermeneutik. Frankfurt a. M.: Athenäum.

Ebeling, Knut 2000. Freud, die Archäologie, die Moderne. Die archäologische Methode als Antwort auf Nietzsches Repräsentationskritik. Nietzscheforschung (7): 127-140.

Ehrenberg, Alain 2015 [1998]. Das erschöpfte Selbst. 2. Aufl. Frankfurt a. M.: Campus.

Elden, Stuart 2006. Discipline, Health and Madness: Foucault's Le pouvoir psychiatrique. History of the Human Sciences (19/1): 39-66.

Eribon, Didier. 1999 [1989]. Michel Foucault. Eine Biographie. Frankfurt a. M.: Suhrkamp.

Eribon, Didier 2016 [2009]. Rückkehr nach Reims. Frankfurt a. M.: Suhrkamp.

Fauvel, Aude 2015. „Ausserhalb der Mauern“ - Für eine neue Geschichte der Irrenanstalten in der Moderne. Therapeutische Umschau (72): 429-435.

Fink-Eitel, Hinrich 1997. Michel Foucault zur Einführung. Hamburg: Junius.

Foucault, Michel 1954. Maladie mentale et personnalité. 1. Aufl. Paris: P.U.F.

Foucault, Michel 1968 [1962]. Psychologie und Geisteskrankheit. 2. Aufl. Frankfurt a. M.: Suhrkamp.

Foucault, Michel 1969 [1961]. Wahnsinn und Gesellschaft. Eine Geschichte des Wahns im Zeitalter der Vernunft. Frankfurt a. M.: Suhrkamp.

Foucault, Michel (Hg.) 1975 [1973]. Der Fall Rivière. Materialien zum Verhältnis von Psychiatrie und Strafjustiz. Frankfurt a. M.: Suhrkamp.

Foucault, Michel 1974 [1966]. Die Ordnung der Dinge. Eine Archäologie der Humanwissenschaften. Frankfurt a. M.: Suhrkamp.

Foucault, Michel 1977 [1971]. Die Ordnung des Diskurses. Frankfurt a. M.: Ullstein.

Foucault, Michel 1977 [1975]. Überwachen und Strafen. Die Geburt des Gefängnisses. Frankfurt a. M.: Suhrkamp.

Foucault, Michel 1981 [1969]. Archäologie des Wissens. Frankfurt a. M.: Suhrkamp.

Foucault, Michel 1983 [1976]. Der Wille zum Wissen. Sexualität und Wahrheit. Bd. 1. Frankfurt a. M.: Suhrkamp.

Foucault, Michel 1988 [1963]: Die Geburt der Klinik. Eine Archäologie des ärztlichen Blicks. Frankfurt a. M.: Suhrkamp.

Foucault, Michel 1988 [1969]. Was ist ein Autor? In: Ders. Schriften zur Literatur. Frankfurt a. M.: Fischer: 7-31.

Foucault, Michel 1989 [1963]. Raymond Roussel. Frankfurt a. M.: Suhrkamp.

Foucault, Michel 2001 [1954]. Einführung [zu Traum und Existenz von L. Binswanger]. In: Ders. Schriften in vier Bänden. Bd. 1. Frankfurt a. M.: Suhrkamp: 107-174.

Foucault, Michel 2001 [1957a]. Die Psychologie von 1850 bis 1950. In: Ders. Schriften in vier Bänden. Bd. 1. Frankfurt a. M.: Suhrkamp: 175-195.

Foucault, Michel 2001 [1957b]. Die wissenschaftliche Forschung und die Psychologie. In: Ders. Schriften in vier Bänden. Bd. 1. Frankfurt a. M.: Suhrkamp: 196-222. 
Foucault, Michel 2001 [1962a]. Einführung [in J.-J. Rousseaus Dialogues]. In: Ders. Schriften in vier Bänden. Bd. 1. Frankfurt a. M.: Suhrkamp: 241-262.

Foucault, Michel 2001 [1964]. Der Wahnsinn, Abwesenheit eines Werkes. In: Ders. Schriften in vier Bänden. Bd. 1. Frankfurt a. M.: Suhrkamp: 539-550.

Foucault, Michel 2001a [1977]. Das Leben der infamen Menschen. Berlin: Merve.

Foucault, Michel 2002 [1972]. Die große Einsperrung. In: Ders. Schriften in vier Bänden. Bd. 2. Frankfurt a. M.: Suhrkamp: 367-382.

Foucault, Michel 2003 [1976]. Hexerei und Wahnsinn. In: Ders. Schriften in vier Bänden. Bd. 3. Frankfurt a. M.: Suhrkamp: 119-123.

Foucault, Michel 2005 [1984]. Was ist Aufklärung? In: Ders. Schriften in vier Bänden. Bd. 4. Frankfurt a. M.: Suhrkamp: 837-848.

Foucault, Michel 2005 [2003]. Die Macht der Psychiatrie. Vorlesungen am Collège de France 1973-1974. Frankfurt a. M.: Suhrkamp.

Foucault, Michel 2006 [1961]. History of Madness. London/New York: Routledge.

Foucault, Michel 2007 [1999]. Die Anormalen. Vorlesungen am Collège de France 1974-1975. Frankfurt a. M.: Suhrkamp.

Freud, Sigmund 1900. Die Traumdeutung. Leipzig/Wien: Franz Deuticke.

Fulford, Kenneth W. M., Martin Davies, Richard Gipps, George Graham, John Sadler, Giovanni Stanghellini und Tim Thornton (Hg.) 2013. The Oxford Handbook of Philosophy and Psychiatry. Oxford: Oxford University Press.

Gahr, Manfred und Maurice Cabanis 2014. Pharmakologisches Neuroenhancement als Erscheinungsform von Michel Foucaults Biomacht. Nervenheilkunde (33): 75-86.

Geisenhanslüke, Achim 2008. Wahnsinn und Gesellschaft. In: Clemens Kammler, Rolf Parr und Ulrich-Johannes Schneider (Hg.). Foucault-Handbuch. Stuttgart: Metzler: 18-31.

Geisenhanslüke, Achim 1997. Foucault und die Literatur. Eine diskurskritische Untersuchung. Opladen: Westdeutscher Verlag.

Gondek, Hans-Dietrich 2008. Schriften zur Psychologie und Geisteskrankheit. In: Clemens Kammler, Rolf Parr und Ulrich-Johannes Schneider (Hg.). Foucault-Handbuch. Stuttgart: Metzler: 12-18.

Gordon, Colin 1990. Histoire de la folie: An unknown Book by Michel Foucault. History of the Human Sciences (3/1): 3-26.

Gordon, Colin 2007. Extreme Prejudice: Notes on Andrew's Scull's TLS Review of Foucault's History of Madness. URL: https://foucaultblog.wordpress.com/2007/05/20/extremeprejudice/ (12.12.2016).

Habermas, Jürgen 1985. Der philosophische Diskurs der Moderne. Frankfurt a. M.: Suhrkamp. Hacking, Ian 2011. Déraison. History of the Human Sciences (24): 14-24.

Hacking, Ian 2004. Between Michel Foucault and Erving Goffman: Between Discourse in the Abstract and face-to-face Interaction. Economy and Society (33): 277-302.

Han, Béatrice 2002. Foucault's Critical Project. Stanford: Stanford University Press.

Hegel, Georg Wilhelm Friedrich 1992 [1830]. Enzyklopädie der philosophischen Wissenschaften im Grundrisse. Hamburg: Meiner.

Heinze, Martin 2008. Psychiatrie. In: Clemens Kammler, Rolf Parr und Ulrich-Johannes Schneider (Hg.). Foucault-Handbuch. Stuttgart: Metzler: 417-426.

Hirsch, Stefan 2010. „Ein ständiges Prinzip der Unruhe." Foucault und die Psychoanalyse. Freie Assoziation - Zeitschrift für das Unbewusste in Organisation und Kultur (13/4): $5-51$.

Honneth, Axel und Martin Saar (Hg.) 2002. Michel Foucault. Zwischenbilanz einer Rezeption. Frankfurt a. M.: Suhrkamp.

Iliopoulos, John 2012. Foucault's Notion of Power and Current Psychiatric Practice. Philosophy, Psychiatry E Psychology (19): 49-58.

James, Seferin 2011. Derrida, Foucault and "Madness, the Absence of an Oeuvre". Meta Research in Hermeneutics, Phenomenology, and Practical Philosophy (3): 379-403.

Jones, Colin und Roy Porter (Hg.) 1994. Reassessing Foucault: Power, Medicine and the Body. London/New York: Routledge.

Joranger, Line 2016. Individual Perception and Cultural Development: Foucault's 1954 Approach to Mental Illness and its History. History of Psychology (19): 40-51.

Kammler, Clemens 2008. Einführung: Konzeptualisierungen der Werke Foucaults. In: Clemens Kammler, Rolf Parr und Ulrich-Johannes Schneider (Hg.). Foucault-Handbuch. Stuttgart: Metzler: 9-11. 
Kammler, Clemens, Rolf Parr und Ulrich-Johannes Schneider (Hg.) 2008. Foucault-Handbuch. Leben - Werk - Wirkung. Stuttgart: Metzler.

Kasten, Ingrid 1992. „Narrheit“ und „Wahnsinn“. Michel Foucaults Rezeption von Sebastian Brants "Narrenschiff“. In: Johannes Janota, Paul Sappler und Frieder Schanze (Hg.). Festschrift für Walter Haug und Burghart Wachinger. Bd. 1. Tübingen: Niemeyer: 233-254.

Kisker, Karl Peter 1979. Antipsychiatrie. In: Karl Peter Kisker, Joachim-Ernst Meyer, Christian Müller und Erik Strömgren (Hg.). Psychiatrie der Gegenwart - Forschung und Praxis. Bd. 1/1. Berlin/Heidelberg: Springer: 811-825.

Kollek, Regine und Thomas Lemke 2008. Der medizinische Blick in die Zukunft. Gesellschaftliche Implikationen prädiktiver Gentests. Frankfurt a. M./New York: Campus.

Koopman, Colin (Hg.) 2011. Special issue: Foucault Across the Disciplines. History of the Human Sciences (24).

Lacan, Jacques 1997 [1955/56]. Die Psychosen. Weinheim/Berlin: Quadriga.

Lagrange, Jacques 2005 [2003]. Situierung der Vorlesungen. In: Michel Foucault: Die Macht der Psychiatrie. Frankfurt a. M.: Suhrkamp: 505-534.

Leferink, Klaus 1997. Sympathie mit der Schizophrenie. In: Manfred Zaumseil und Klaus Leferink (Hg.). Schizophrenie der Moderne - Modernisierung der Schizophrenie. Bonn: Psychiatrie Verlag: 27-81.

Leibbrand, Werner 1964. Das Geschichtswerk Michel Foucaults. Sudhoffs Archiv für Geschichte der Medizin und der Naturwissenschaften (48): 353-359.

Lemke, Thomas 2011. Eine Kritik der politischen Vernunft - Foucaults Analyse der modernen Gouvernementalität. Hamburg/Berlin: Argument.

Leoni, Federico 2013. From Madness to Mental Illness: Psychiatry and Biopolitics in Michel Foucault. In: Kenneth W. M. Fulford, Martin Davies, Richard Gipps, George Graham, John Sadler, Giovanni Stanghellini und Tim Thornton (Hg.). The Oxford Handbook of Philosophy and Psychiatry. Oxford: Oxford University Press: 85-98.

Macherey, Pierre 1986. Aux Sources de L'Histoire de la Folie: une rectification et ses limites. Critique (43/471-472): 753-774.

Matthews, Eric 1995. Moralist or Therapist? Foucault and the Critique of Psychiatry. Philosophy, Psychiatry \& Psychology (2): 19-30.

May, Todd 2005. Foucault's Relation to Phenomenology. In: Gary Cutting (Hg.). The Cambridge Companion to Foucault. 2. Aufl. Cambridge: Cambridge University Press: 284-311.

Mazumdar, Pravu 2008. Der archäologische Zirkel. Zur Ontologie der Sprache in Michel Foucaults Geschichte des Wissens. Bielefeld: transcript.

Midelfort, H. C. Erik 1999. A History of Madness in Sixteenth-Century Germany. Stanford: Stanford University Press.

Moreau, Jacques-Joseph 1845. Du haschisch et de l'aliénation mentale, études psychologiques. Paris: Éditions Fortin.

Nietzsche, Friedrich 1999 [1887]. Genealogie der Moral. In: Ders.: Kritische Studienausgabe. Bd. 5. München: dtv: 245-412.

Novella, Enric 2008. Der junge Foucault und die Psychopathologie: Psychiatrie und Psychologie im frühen Werk von Michel Foucault. Berlin: Logos.

Paltrinieri, Luca 2015. De quelques sources de ,Maladie mentale et personnalité - Réflexologie pavlovienne et critique sociale. In: Jean-François Bert und Elisabetta Basso (Hg.). Foucault à Münsterlingen: A l'origine de l'Histoire de la folie. Paris: Editions de l'Ecole des Hautes Etudes en Sciences Sociales: 197-219.

Peters, Timothy J. und Allan Beveridge (2010). The Blindness, Deafness and Madness of King George III: Psychiatric Interactions. Journal of the Royal College of Physicians of Edinburgh (40): 81-85.

Pinel, Philippe 1801 [1800]. Philosophisch-medicinische Abhandlung über Geistesverwirrungen oder Manie. Wien: Schaumburg.

Philo, Chris 2007. Review Essay: Michel Foucault, Psychiatric Power. Foucault Studies (4): 149-163.

Porter, Roy 1985. The Patient's View: Doing Medical History from Below. Theory and Society (14): 175-198. 
Porter, Roy 1987. Mind-Forg'd Manacles. A History of Madness in England from the Restoration to the Regency. London: Athlone.

Riccardi, Mattia 2014. Nietzsche und die Erkenntnistheorie und Metaphysik. In: Helmut Heit und Lisa Heller (Hg.). Handbuch Nietzsche und die Wissenschaften. Berlin/Boston: de Gruyter: 242-264.

Raffnsøe, Sverre, Morten S. Thaning und Marius Gudmand-Hoyer 2015. Michel Foucault: A Research Companion. Basingstoke: Palgrave Macmillan.

Rüb, Matthias 1990. Das Subjekt und sein Anderes: Zur Konzeption von Subjektivität beim frühen Foucault. In: Eva Erdmann, Rainer Forst und Axel Honneth (Hg.). Ethos der Moderne. Foucaults Kritik der Aufklärung. Frankfurt a. M./New York: Campus: 187-201.

Rose, Nikolas 2006. Disorders Without Borders? The Expanding Scope of Psychiatric Practice. BioSocieties (1): 465-484.

Sachße, Christoph und Florian Tennstedt 1998. Geschichte der Armenfürsorge in Deutschland. Bd. 1: Vom Spätmittelalter bis zum 1. Weltkrieg. 2. Aufl. Stuttgart: Kohlhammer.

Sartre, Jean-Paul 1971 [1940]. Das Imaginäre. Reinbek: Rowohlt.

Scull, Andrew 2007. The Fictions of Foucault's Scholarship. Times Literary Supplement, 21. März 2007: 3-4.

Scull, Andrew 2015. Madness in Civilization: A Cultural History of Insanity from the Bible to Freud, from the Madhouse to Modern Medicine. London: Thames and Hudson.

Schmiedebach, Heinz-Peter 2016. Entgrenzungsphänomene des Wahnsinns - Einleitung. In: Ders. Entgrenzungen des Wahnsinns. Psychopathie und Psychopathologisierungen um 1900. Berlin/Boston: De Gruyter: 1-28.

Segre, Sandro 2014. Contemporary Sociological Thinkers and Theories. Farnham/Burlington: Ashgate.

Still, Arthur und Irving Velody (Hg.) 1992. Rewriting the History of Madness. Studies in Foucault's 'Histoire de la folie'. London/New York: Routledge.

Stolberg, Michael 2012. Möglichkeiten und Grenzen einer retrospektiven Diagnose. In: Waltraud Pulz (Hg.). Zwischen Himmel und Erde. Körperliche Zeichen der Heiligkeit. Stuttgart: Steiner: 209-227.

Thoma, Samuel 2016. Le tragique et la folie. In: Olivier Frérot und Chris Younès (Hg.). $A$ l'épreuve d'exister avec Henri Maldiney. Paris: Hermann: 177-190.

Unterthurner, Gerhard 2007. Foucaults Archäologie und Kritik der Erfahrung. Wien: Turia + Kant.

Wellbery, David E. 2007. Form und Funktion der Tragödie nach Nietzsche. In: Bettine Menke und Christoph Menke (Hg.). Tragödie. Trauerspiel. Spektakel. Berlin: Theater der Zeit: 199-212.

Wernli, Martina 2014. Schreiben am Rand. Die „Bernische kantonale Irrenanstalt Waldau“ und ihre Narrative (1895-1936). Bielefeld: transcript.

Woods, Angela 2011. The Sublime Object of Psychiatry. Schizophrenia in Clinical and Cultural Theory. Oxford/New York: Oxford University Press.

\author{
Burkhart Brückner \\ Fachbereich Sozialwesen \\ Hochschule Niederrhein - University of Applied Sciences \\ Richard-Wagner-Str. 101 \\ 41065, Mönchengladbach \\ Deutschland \\ burkhart.brueckner@hs-niederrhein.de
}


Lukas Iwer

Sektion phänomenologische Psychopathologie und Psychotherapie Universität Heidelberg, Klinik für Allgemeine Psychiatrie

Voßstr. 4

69115, Heidelberg

Deutschland

Samuel Thoma

Sektion phänomenologische Psychopathologie und Psychotherapie Universität Heidelberg, Klinik für Allgemeine Psychiatrie

Voßstr. 4

69115, Heidelberg

Deutschland 